\title{
Nanotechnology in cancer therapy: An overview and perspectives (Review)
}

\author{
Sarishti Sharma ${ }^{1}$, Anita Bhatia ${ }^{2 *}$, Neeru Gakkhar ${ }^{3}$ \\ ${ }^{1}$ Research Scholar, ${ }^{2}$ Associate Professor, ${ }^{3}$ Assistant Professor, ${ }^{1}$ Kota College of pharmacy, Kota, Rajasthan, ${ }^{2,3}$ S.S. Jain \\ Subodh P.G. Autonomous College, University of Rajasthan, Jaipur, Rajasthan, India \\ *Corresponding Author: Anita Bhatia \\ Email: sharmasarishti09@gmail.com
}

\begin{abstract}
Nanotechnology is the study and use of structures between 1 nanometer and 100 nanometers in size. Cancer is the leading cause of death among people younger than 85 years. Nanoparticles that deliver chemotherapy drugs directly to cancer cells are under development. Nanomaterials are increasingly used as drug carriers for cancer therapy. Nanomaterials also appeal to researchers in the areas of cancer diagnosis and biomarker discovery. Among the newly developed nanomedicine and nanodevices such as quantum dots, nanowires, nanotubes, nanocantilevers, and nanopores, nanoshells and nanoparticles are the most promising applications for various cancer treatments. This article aims at giving an overview of the present status of nanotechnology in cancer therapy and the prospects of nanomaterials as drug carriers for future clinical applications are also discussed.
\end{abstract}

Keywords: Nanotechnology, Nanomedicine, Application of nanomedicine, Nanomedicine for cancer, Risks of nanotechnology.

\section{Introduction}

The term "nanotechnology" is a concept that has only emerged in the last decade with the prefix "nano" cited from the Greek word "nanos", indicating that something is dwarf-sized. Therefore, the term "nanotechnology" refers to a technology that uses very small particles invisible to the naked eye. Before the century, although the term nanotechnology had not yet been globally defined, the applications of nanotechnology were already used in the industrial field. ${ }^{1}$

Cancer is one of the main causes of death worldwide and, according to the World Health Organization; the number of cancer-related deaths is going to increase up to approximately 13.2 million people a year by 2030. Currently, cancer treatments rely on chemotherapy, radiotherapy, and surgery. Unfortunately, these approaches are not specific, since they can attack both tumor and healthy tissues, causing adverse side effects to already debilitated patients (e.g., nausea, hair loss, weakness, and immunodepression). Aggressive tumors proliferate by creating new vessels in the surrounding tissues through a process termed angiogenesis. This vasculature shows several abnormalities in the number of endothelial cells and tridimensional structure, and the gaps between neighboring cells are larger than in physiological conditions, resulting in enhanced permeability. Moreover, tumor tissues lack an efficient lymphatic drainage system. All these phenomena are at the origin of the "enhanced permeability and retention" (EPR) effect, thanks to which some drugs can accumulate more easily around tumor tissues with respect to healthy ones. However, due to the augmented pressure at the core of the tumor mass, common drugs penetrate with many difficulties and are mostly retained at the periphery. ${ }^{2,3}$

For these reasons, there is an increasing and urgent need for designing new tools capable of improving diagnosis and reducing the severe reactions correlated with conventional therapies. Recently, researchers are putting a lot of effort in creating drugs that univocally target cancer cells and are highly bioavailable, in order to decrease the administration doses and to prevent undesired cytotoxicity and drug resistance. In the last two decades, a branch of nanotechnology, namely nanomedicine, emerged as an innovative way to exploit nanomaterials for human health, including cancer treatment. Nanomedicine allows performing early diagnosis, curing with minimal side effects, and 
evaluating the efficacy of the treatments in a noninvasive way.

In this review, we will provide an overview of the main nanoparticles that are currently tested for potential exploitation in nanomedicine; applications to biological models and their current status in the clinical context will be also described, highlighting their impact as cancer nanotheranostic agents.

\section{What is Nanoparticles?}

Nanoparticle are particles between 1 and 100 nanometres $(\mathrm{nm})$ in size with a surrounding interfacial layer. The interfacial layer is an integral part of nanoscale matter, fundamentally affecting all of its properties. The interfacial layer typically consists of ions, inorganic and organic molecules. Organic molecules coating inorganic nanoparticles are known as stabilizers, capping and surface ligands, or passivating agents. ${ }^{4,5}$

In nanotechnology, a particle is defined as a small object that behaves as a whole unit with respect to its transport and properties. Particles are further classified according to diameter. In recent decades the development of microscopes capable of displaying particles as small as atoms has allowed scientists to see what they are working with. In fact, nanoparticles embrace a wide spectrum of applications sparking another industrial revolution.

The nanoparticles are of different shape, size and structure. It be spherical, cylindrical, tubular, conical, hollow core, spiral, flat, etc. or irregular and differ from $1 \mathrm{~nm}$ to $100 \mathrm{~nm}$ in size. The surface can be a uniform or irregular with surface variations. Some nanoparticles are crystalline or amorphous with single or multi crystal solids either loose or agglomerated.

Numerous synthesis methods are either being developed or improved to enhance the properties and reduce the production costs. Some methods are modified to achieve process specific nanoparticles to increase their optical, mechanical, physical and chemical properties. A vast development in the instrumentation has led to an improved nanoparticle characterisation and subsequent application

\section{Classification of Nanoparticles}

The nanoparticles are generally classified into the organic, inorganic and carbon based. ${ }^{6}$

\section{Organic Nanoparticles}

Dendrimers, micelles, liposomes and ferritin, etc. are commonly knows the organic nanoparticles or polymers. These nanoparticles are biodegradable, nontoxic, and some particles such as micelles and liposomes has a hollow core, also known as nanocapsules and are sensitive to thermal and electromagnetic radiation such as heat and light. These unique characteristics make them an ideal choice for drug delivery. The organic nanoparticles are most widely used in the biomedical field for example drug delivery system as they are efficient and also can be injected on specific parts of the body that is also known as targeted drug delivery.

\section{Inorganic Nanoparticles}

Inorganic nanoparticles are particles that are not made up of carbon. Metal and metal oxide based nanoparticles are generally categorised as inorganic nanoparticles.

\section{Metal based}

Nanoparticles that are synthesised from metals to nanometric sizes either by destructive or constructive methods are metal based nanoparticles. Almost all the metals can be synthesised into their nanoparticles. ${ }^{9}$ The commonly used metals for nanoparticle synthesis are aluminium $(\mathrm{Al})$, cadmium $(\mathrm{Cd})$, cobalt $(\mathrm{Co})$, copper $(\mathrm{Cu})$, gold $(\mathrm{Au})$, iron $(\mathrm{Fe})$, lead $(\mathrm{Pb})$, silver $(\mathrm{Ag})$ and zinc $(\mathrm{Zn})$. The nanoparticles have distinctive properties such sizes as low as 10 to $100 \mathrm{~nm}$, surface characteristics like high surface area to volume ratio, pore size, surface charge and surface charge density, crystalline and amorphous structures, shapes like spherical and cylindrical and colour, reactivity and sensitivity to environmental factors such as air, moisture, heat and sunlight etc.

\section{Metal oxides based}

The metal oxide based nanoparticles are synthesised to modify the properties of their respective metal based nanoparticles, for example nanoparticles of iron $(\mathrm{Fe})$ 
instantly oxidises to iron oxide $\left(\mathrm{Fe}_{2} \mathrm{O}_{3}\right)$ in the presence of oxygen at room temperature that increases its reactivity compared to iron nanoparticles. Metal oxide nanoparticles are synthesised mainly due to their increased reactivity and efficiency. The commonly synthesised are Aluminium oxide $\left(\mathrm{Al}_{2} \mathrm{O}_{3}\right)$, Cerium oxide $\left(\mathrm{CeO}_{2}\right)$, Iron oxide $\left(\mathrm{Fe}_{2} \mathrm{O}_{3}\right)$, Magnetite $\left(\mathrm{Fe}_{3} \mathrm{O}_{4}\right)$, Silicon dioxide $\left(\mathrm{SiO}_{2}\right)$, Titanium oxide $\left(\mathrm{TiO}_{2}\right)$, Zinc oxide $(\mathrm{ZnO})$. These nanoparticles have possessed exceptional properties when compared to their metal counterparts.

\section{Carbon based}

The nanoparticles made completely of carbon are knows as carbon based. They can be classified into fullerenes, graphene, carbon nano tubes (CNT), carbon nanofibers and carbon black and sometimes activated carbon in nano size.

1. Fullerenes: Fullerenes (C60) is a carbon molecule that is spherical in shape and made up of carbon atoms held together by sp hybridization. About 28 to 1500 carbon atoms forms the spherical structure with diameters up to $8.2 \mathrm{~nm}$ for a single layer and 4 to $36 \mathrm{~nm}$ for multi-layered fullerenes.

2. Graphene: Graphene is an allotrope of carbon. Graphene is a hexagonal network of honeycomb lattice made up of carbon atoms in a two dimensional planar surface. Generally the thickness of the graphene sheet is around $1 \mathrm{~nm}$.

3. Carbon Nano Tubes (CNT): Carbon Nano Tubes (CNT), a graphene nanofoil with a honeycomb lattice of carbon atoms is wound into hollow cylinders to form nanotubes of diameters as low as $0.7 \mathrm{~nm}$ for a single layered and $100 \mathrm{~nm}$ for multi-layered CNT and length varying from a few micrometres to several millimetres. The ends can either be hollow or closed by a half fullerene molecule.

4. Carbon Nanofiber: The same graphene nanofoils are used to produce carbon nanofiber as CNT but wound into a cone or cup shape instead of a regular cylindrical tubes.

5. Carbon black: An amorphous material made up of carbon, generally spherical in shape with diameters from 20 to $70 \mathrm{~nm}$. The interaction between the particles are so high that they bound in aggregates and around $500 \mathrm{~nm}$ agglomerates are formed.

\section{Advantages of Nanoparticles}

1. Reduction in fed/fasted variability

2. Dose proportionality

3. Decreased toxicity

4. Increased bioavailability

5. Smaller dosage form (i.e., smaller tablet)

6. Stable dosage forms of drugs which are either unstable or have unacceptably low bioavailability in non-nanoparticulate dosage forms

\section{Applications of Nanotechnology}

The 2000s have seen the beginnings of the applications of nanotechnology in commercial products, although most applications are limited to the bulk use of passive nanomaterials. ${ }^{10}$ Examples include:

1. Titanium dioxide and zinc oxide nanoparticles in sunscreen, cosmetics and some food products

2. Silver nanoparticles in food packaging, clothing, disinfectants and household appliances.

3. Silver Nano; carbon nanotubes for stain-resistant textiles; and cerium oxide as a fuel catalyst.

Nanotechnology is being used in developing countries to help treat disease and prevent health issues. The umbrella term for this kind of nanotechnology is Nanomedicine.

1. Nanotechnology is also being applied to or developed for application to a variety of industrial and purification processes. Purification and environment cleanup applications include the desalination of water, water filtration, filtration, wastewater treatment, groundwater treatment, and other nanoremediation.

2. In industry, applications may include construction materials, military goods, and nano-machining of nano-wires, nano-rods, few layers of graphene, ${ }^{3}$ etc.

3. Also, recently a new field arisen from the root of Nanotechnology is called Nanobiotechnology. Nanobiotechnology is the biology-based, application-oriented frontier area of research in the 
hybrid discipline of Nanoscience and biotechnology with an equivalent contribution.

\section{Nanotechnology in cancer therapy}

Nanomaterials are increasingly used as drug carriers for cancer therapy. Nanomaterials also appeal to researchers in the areas of cancer diagnosis and biomarker discovery. Several antitumor nanodrugs are currently being tested in preclinical and clinical trials and show promise in therapeutic and other settings. We review the development of nanomaterial drug carriers, including liposomes, polymer nanoparticles, dendritic polymers, and nanomicelles, for the diagnosis and treatment of various cancers. ${ }^{11,12}$

\section{Nanotechnology to fight and cure cancer}

Cancer is one of the leading causes of death in the world and remains a difficult disease to treat. Current problems associated with conventional cancer chemotherapies include insolubility of drugs in aqueous medium; delivery of sub-therapeutic doses to target cells; lack of bioavailability; and most importantly, non-specific toxicity to normal tissues. Recent contributions of nanotechnology research address possible solutions to these conundrums. Nevertheless, challenges remain with respect to delivery to specific sites, real time tracking of the delivery system, and control over the release system after the drug has been transported to the target site.

Nanotechnology applications to diagnose and treat cancers are already a reality providing a wide range of new tools and possibilities, from earlier diagnostics and improved imaging to better, more efficient, and more targeted therapies.

\section{Cancer Diagnostics}

More lives could be saved by early detection of cancer than by any form of treatment at advanced stages. Circulating tumor cells (CTCs), which are viable cells derived from tumors, are hypothesized to represent the origin of metastatic disease.

Nanotechnology can be used to develop devices that indicate when those markers appear in the body and that deliver agents to reverse premalignant changes or to kill those cells that have the potential to become malignant.

\section{Nanotechnology in Cancer Treatment}

Nanotechnology cancer treatments may lead to destroying cancer tumors with minimal damage to healthy tissue and organs, as well as the detection and elimination of cancer cells before they form tumors.

Most efforts to improve cancer treatment through nanotechnology are at the research or development stage. However there are many universities and companies around the world working in this area.

\section{Nanotechnology Cancer Treatments; Nanoparticle Chemotherapy}

A targeted chemotherapy treatment under development uses a nanoparticle called CRLX101. The company developing this targeted chemotherapy method is called Cerulean Pharma. Researchers at the University of Georgia are working on a method to fight prostate cancer. They are using nanoparticles to deliver a molecule called IPA-3 to the cancer cells. In laboratory mice studies the IPA-3 appears to reduce the growth of prostate cancer cells.

\section{Nanotechnology Cancer Treatments; Heat}

Another technique being developed works on destroying cancer tumors by applying heat. Nanoparticles called AuroShells absorb infrared light from a laser, turning the light into heat. The company developing this technique is called Nanospectra. Targeted heat therapy is being developed to destroy breast cancer tumors.

In this method antibodies that are strongly attracted to proteins produced in one type of breast cancer cell are attached to nanotubes, causing the nanotubes to accumulate at the tumor. Infrared light from a laser is absorbed by the nanotubes and produces heat that incinerates the tumor.

\section{Nanotechnology Cancer Treatments; Radiation}

Researchers have developed nanoparticles containing a radioactive core with attached molecules that attach to lymphoma tumor cells. The researchers are designing this method to stop the spread of cancer from the primary tumor. Researchers are investigating the use of bismuth nanoparticles to concentrate radiation used in radiation therapy to treat cancer tumors. Initial results indicate that the bismuth 
nanoparticles would increase the radiation dose to the tumor by 90 percent.

A method to make radiation therapy more effect in fighting prostate cancer is using radioactive gold nanoparticles attached to a molecule that is attracted to prostate tumor cells. Researchers believe that this method will help concentrate the radioactive nanoparticles at the cancer tumors, allowing treatment of the tumors with minimal damage to healthy tissue.

\section{Nanotechnology Cancer Treatments; Miscellaneous}

Researchers at Tel Aviv University are developing a vaccine for melanomabased using polymer nanoparticles to which melanoma related peptides have been attached. Researchers have demonstrated a nanoparticle that kills lymphoma cancer cells. They use a nanoparticle which looks like HDL cholesterol, but with a gold nanoparicle at it's core. When this nanoparticle attaches to a lymphoma cell it blocks the cancer cell from attaching to real HLD cholesterol, starving the cancer cell.

A method being developed to fight skin cancer uses gold nanoparticles to which RNA molecules are attached. The nanoparticles are in an ointment that is applied to the skin. The nanoparticles penetrate the skin and the RNA attaches to a cancer related gene, stopping the gene from generating proteins that are used in the growth of skin cancer tumors.

\section{Nanomedicine: Future Applications}

Future application of nanomedicine may involve the elimination of bacterial infections in a patient within minutes, instead of using treatment with antibiotics over a period of weeks. In future, by the application of nanomedicine, we may have the ability to perform surgery at the cellular level, removing individual diseased cells and even repairing defective portions of individual cells. It may contribute significantly for lengthening of the human lifespan by repairing cellular level conditions that cause the body to age.

\section{Source of Funding}

None.

\section{Conflict of Interest}

None.

\section{References}

1. JBoer, JitKang Lim, Xin Chen,Magdalena Plebanski, and $\mathrm{R}$ ohimah Mohamud $A$ Perspective Review on the Role of Nanomedicine in the Modulation of TNF-TNFR2 Axis in Breast Cancer Immunotherapy. J Oncology 2019:13.

2. Cristina Riggio, Eleonora Pagni, Vittoria Raffa, and Alfred Cuschieri. Nano-Oncology: Clinical Application for Cancer Therapy and Future Perspectives. J Nanomaterials 2011, Article ID 164506, 10 pages

3. M. Ferrari, "Cancer nanotechnology: opportunities and challenges,” Nature Reviews Cancer, 2005;5(3):161-71.

4. S Wang, Z.L, Ballato J, Foulger, S.H., and Carroll, D.L. $J$ Am Chem Soc 2003;125:16186 -7.

5. van der Zande, B.M.I, B ö hmer, M.R, Fokkink, L.G.J, and Sch ö neberger, C. J Phys Chem B 1997;101; 852-4.

6. Custódio C, Santo V, Oliveira M, Gomes M, Reis R, Mano J. Functionalized microparticles producing scaffolds in combination with cells. Adv Funct Mater 2014;24(10):1391-1400.

7. Lima A, Puga A, Mano J, Concheiro A, Alvarez-Lorenzo C. Free and copolymerized $\gamma$-cyclodextrins regulate the performance of dexamethasone-loaded dextran microspheres for bone regeneration. J Mater Chem B 2014;2(30):4943-56.

8. Simón-Yarza T, Formiga F, Tamayo E, Pelacho B, Prosper F, Blanco-Prieto M. PEGylated-PLGA microparticles containing VEGF for long term drug delivery. Int J Pharm 2013;440(1):13-8.

9. V. Mody, R. Siwale, A. Singh, and H. R. Mody, "Introduction to metallic nanoparticles," J Pharm Bioallied Sci 2010;2:282-9.

10. PT Edward; MS Michele. Application of nanotechnology: A case study in the pharmaceutical area. 2004.

11. Farokhzad OC, Langer R. Impact of nanotechnology on drug delivery. ACS Nano 2009;3:16-20.

12. Green MR, Manikhas GM, Orlov S, Afanasyev B, Makhson AM, Bhar P et al. Abraxane, a novel Cremophorfree, albumin-bound particle form of paclitaxel for the treatment of advanced non-small-cell lung cancer. Ann Oncol 2006;17:1263-8.

How to cite this article: Sharma S, Bhatia A, Gakkhar N. Nanotechnology in cancer therapy: An overview and perspectives (Review). Int $J$ Pharm Chem Anal 2019;6(4):110-4. 